\title{
Relationship Between Anxiety, Depression, and Quality of Life in Adult Patients With Cystic Fibrosis
}

\author{
Abebaw Mengistu Yohannes PhD, Thomas George Willgoss MSc, Francis Ade Fatoye PhD, \\ Mary Dodd Dip PT, and Kevin Webb MD
}

\begin{abstract}
BACKGROUND: The impact of anxiety and depression on quality of life (QOL) in adult patients with cystic fibrosis (CF) is fully unknown. We investigated the prevalence and factors associated with anxiety and depression, including QOL, in adult CF patients. METHODS: One hundred twenty-one adult CF subjects, age $\geq 18$ years were recruited from our out-patient clinic. Participants self-completed the Hospital Anxiety Depression Scale and the Cystic Fibrosis Quality of Life Questionnaire (CF-QOL). Socio-demographic data and values for lung function were extracted from the medical notes. RESULTS: Mean \pm SD age was $30 \pm 8.8$ years, and age ranged 18 70 years. Forty $(33 \%)$ were identified with anxiety symptoms, $20(17 \%)$ with depressive symptoms. Factors related with depression were impaired QOL and low lung function. Anxiety was associated with difficulty in interpersonal relationships and severity of chest symptoms. The CF-QOL subdomains (physical functioning, social functioning, treatment issues, chest symptoms, emotional functioning, concerns for the future, interpersonal relationships, body image, future/career concerns, and total CF-QOL) were all significantly correlated with anxiety $(P<.001)$ and with depression $(P<.001)$, respectively. CONCLUSIONS: Anxiety and depressive symptoms are common in adult CF patients. They are associated with poorer QOL, low lung function, reduced physical functioning, and severity of chest symptoms. Therefore, routine screening for symptoms of anxiety and depression is a worthy endeavor, and those identified with elevated clinical symptoms should be referred to receive appropriate treatment. Key words: cystic fibrosis; anxiety; depression; quality of life; rehospitalization. [Respir Care 2012;57(4):550-556. ( 2012 Daedalus Enterprises]
\end{abstract}

\section{Introduction}

Cystic fibrosis (CF) is an autosomal recessive clinical condition characterized by $\mathrm{CF}$ transmembrane conductance

\footnotetext{
Dr Yohannes, Mr Willgoss, and Dr Fatoye are affiliated with the Department of Health Professions and the Research Institute for Health and Social Change, Manchester Metropolitan University, Manchester, United Kingdom. Ms Dodd and Dr Webb are affiliated with the Cystic Fibrosis Unit, Wythenshawe Hospital, University of Manchester, Manchester, United Kingdom.
}

This research was partly supported by a grant from the Research Institute for Health and Social Change, Manchester Metropolitan University, Manchester, United Kingdom. The authors have disclosed no conflicts of interest.

Correspondence: Abebaw Mengistu Yohannes PhD, Department of Health Professions, Manchester Metropolitan University, Elizabeth Gaskell Campus, Hathersage Road, Manchester, United Kingdom M13 0JA. E-mail: a.yohannes@mmu.ac.uk.

DOI: $10.4187 /$ respcare. 01328 regulator dysfunction. ${ }^{1} \mathrm{CF}$ is a life threatening and incurable disease. It is estimated to be diagnosed 1 in 2,0003,000 in white newborns. ${ }^{2,3}$ It is a severe and progressive disease characterized by decreased physical activities and excessive dyspnea on exertion. ${ }^{3}$ Recent advances in the detection and management of $\mathrm{CF}$ have led to an increase in the number of individuals surviving into adulthood, and as a result, patients with $\mathrm{CF}$ are living with a chronic illness with a substantial psychological and social challenge., ${ }^{4,5}$

A few studies have examined the prevalence of anxiety and depression in subjects with CF. Havermans and colleagues ${ }^{6}$ reported normal scores for anxiety and depression using the Hospital Anxiety and Depression Scale (HADS) among 57 adult CF subjects. However, although average scores were normal, $30 \%$ of CF subjects scored with anxiety and $13 \%$ with depressive symptoms. These data suggest that although average scores may be normal, the prevalence of clinical anxiety and depression may be elevated in patients with $\mathrm{CF}$. 
A recent epidemiological ${ }^{7}$ study of 670 adolescents and adults with CF found that $20.6 \%$ of patients had anxiety symptoms and $9.6 \%$ had depressive symptoms. These findings were compared against data from a large national survey of healthy subjects (anxiety symptoms $6.7 \%$ and depressive symptoms 5.2\%); it was found that the prevalence of anxiety was significantly higher in patients with $\mathrm{CF}$ compared with the general population. In addition, a recent study of 76 adults with $\mathrm{CF}$ found that $30 \%$ screened positive for depression using the Beck Depression Inventory, with over half of these falling into the moderatesevere category for depression. ${ }^{8}$ The discrepancy in these findings may be due to the use of various measurement scales and population samples. However, the HADS was especially designed for medical populations and did not include somatic questions (eg, fatigue) that potentially can be confounded with a medical condition. Therefore, further research into prevalence of anxiety and depression is warranted, particularly in a larger sample of CF patients.

The development of disease-specific CF Quality of Life (CF-QOL) measures in the past few decades has enabled valid and reliable measurement of this multi-dimensional concept. ${ }^{9,10}$ However, the factors that contribute to health related quality of life (HRQOL) in patients with $\mathrm{CF}$ are unclear. A recent study that examined the impact of disease severity ${ }^{11}$ on HRQOL found only modest relationships. The impact of anxiety and depression in relation to quality of life in CF patients is not fully known.

A recent small study ${ }^{6}(n=57)$ found that after controlling for lung function, subjects who reported symptoms of anxiety had poorer HRQOL scores in areas including emotional functioning and vitality as measured on the Dutch version of the teen/adult CF Questionnaire (CF-14+). In addition, subjects who reported symptoms of depression had poorer scores for dimensions of eating disturbances, emotional functioning, and body image. Riekert and colleagues $^{8}(n=76)$ found that depressive symptoms were associated with poorer HRQOL across all dimensions. These findings suggest that there may be a relationship between comorbid anxiety, depression, and HRQOL, but both studies were limited by their small sample sizes.

To date, prevalence and factors that are related to anxiety and depression have not been extensively investigated in adult patients with CF. Anxiety and depression may lead to impaired quality of life and lower adherence to treatment. ${ }^{12}$ Therefore, understanding the prevalence and impact of anxiety and depressive symptoms on HRQOL in patients with CF may help clinicians to develop appropriate and effective management intervention strategies to reduce morbidity and healthcare costs. Thus, the objectives of this study are to explore the prevalence and factors associated with anxiety and depression, including quality of life, in a large cohort of adults with CF.

\section{QUICK LOOK}

\section{Current knowledge}

The impact of anxiety and depression on quality of life in adult patients with cystic fibrosis is not completely understood.

\section{What this paper contributes to our knowledge}

Anxiety and depressive symptoms are common in cystic fibrosis patients. They are associated with poorer quality of life, low lung function, reduced physical functioning, and severity of chest symptoms.

\section{Method}

\section{Sample}

A consecutive series of adults ( $\geq 18$ years) with the diagnosis of $\mathrm{CF}$ were recruited for the study during a routine out-patient visit at a University teaching hospital which covers the North-West of England. CF patients who were experiencing exacerbation or were admitted for treatment in the previous 6 weeks were excluded. There is no standard definition for an exacerbation. Therefore, we have composed an operational definition. An exacerbation is defined as an event in the natural course of the disease characterized by a change in the patient's baseline condition that includes increased cough, increased sputum production, shortness of breath, loss of appetite, loss of weight, and lung function decline, which is beyond normal dayto-day variation, is acute onset, and may warrant a change in regular medication. ${ }^{13,14}$

Subjects were recruited from March 2008 to September 2009. The research assistant identified all patients from the appointment record for their review assessment 2 weeks in advance of their appointment either for their annual assessment review clinic or out-patient appointments. All patients were invited to participate in the study, a patient information sheet that explained the purpose of the study and consent form were sent to patients' home address from the researcher by post. The purpose of the letter was to give sufficient time for the patients to read and discuss the project with their family before attending for their next appointment. The local Research Ethics Committee approved the study.

\section{Procedure}

The research assistant approached the CF patients at the out-patient clinic and explained the purpose of the study. Those interested in participating in the study were asked to 
sign the consent form and to allow the use of their medical records. Participants were given the 2 questionnaires to complete in a random order to assess anxiety, depression, and quality of life while waiting in the out-patient clinic. Demographic data characteristics (age, sex, lung function results, weight, height, comorbidities, medications, and hospital admission in the previous year) were collected from the subject's most recent medical records.

\section{Outcome Measures}

\section{Anxiety and Depression}

Anxiety and depression were assessed using the Hospital Anxiety and Depression Scale (HADS). ${ }^{15}$ This selfscreening tool consists of 14 items: 7 items that measure anxiety, and 7 items that measure depression. Each item is scored on a 4 point Likert scale $(0-3)$ with a higher score indicating more anxiety or depressive symptoms. The minimum score for both scales is 0 and the highest score is 21 . A score of $\geq 8$ indicates probable anxiety or depression, while a score of $\geq 11$ indicated a 'case' for diagnosis of clinical anxiety or depression. The HADS is a reliable and valid scale for patients with medical conditions. ${ }^{15}$ It has been used as a valid screening tool for anxiety and depression in a variety of populations, including primary care patients, psychiatric patients, and the general population. ${ }^{16,17}$

\section{Health-related Quality of Life}

Health-related quality of life was measured with the Cystic Fibrosis Quality of Life Questionnaire (CF-QOL). ${ }^{9}$ The self-completed CF-QOL consists of 52 items across 9 domains of functioning: physical functioning, social functioning, treatment issues, chest symptoms, emotional functioning, concerns for the future, interpersonal relationships, body image, and future/career concerns. Each item is scored on a 6 point Likert scale (1-6), and each domain is scored out of 100, with a higher score of each domain reflecting a better quality of life. The CF-QOL is a validated diseasespecific measure in adults and adolescents with CF. ${ }^{9}$

\section{Data Analysis}

Descriptive statistics were used where appropriate. Comparison between variable means was by the Student $t$ test. We employed a 2-stage process of analyzing the data in order to decrease the possibility of a type 1 error. ${ }^{18}$ In the first stage the relationships between depression, anxiety, quality of life, sex, health status, age, and lung function were examined using the Spearman and Pearson correlation tests to analyze the data where appropriate (according to the distribution of the data). In the second stage, with bivariate analysis, only significantly associated $(P<.10)$ variables were included in a stepwise multiple regression analysis. The regression analysis was performed using an exit level of $P<.05$. Three linear multiple regressions analyses were conducted: to examine factors that determine depression using HAD depression score as the dependent variable; to examine variables that relate to anxiety using the HAD anxiety score as the dependent variable; and to examine factors that determine quality of life using the total CF-QOL score as the dependent variable. Significance was set at $P<.05$. We carried out the data analysis using software (SPSS version 16, SPSS, Chicago, Illinois).

\section{Results}

We approached 125 adult CF patients. Out of these, 121 $\mathrm{CF}$ patients participated in the study. Four CF patients declined to participate in the study ( 2 men and 2 women). The mean $\pm \mathrm{SD}$ age was $30 \pm 8.8$ years, and the age range was $18-70$ years. Mean $\pm \mathrm{SD} \mathrm{FEV}_{1}$ was $2.23 \pm 0.93 \mathrm{~L}$, $\mathrm{FEV}_{1}$ percent of predicted was $47 \pm 11.2 \%$, depression score was $3.6 \pm 3.3$, and anxiety was $6.05 \pm 3.8$. The majority $(75 \%)$ reported comorbid medical illnesses. The most common comorbidities identified in the study include diabetes $(50,41 \%)$, pancreatic insufficiency (39, $32 \%$ ), osteoporosis/osteopenia (25. 21\%), cardiovascular problems including hypertension $(25,21 \%)$, and others (eg, glucose intolerance [7, 6\%]). Most participants had not been hospitalized: $73(60 \%)$ in the previous year prior to initial contact. Table 1 shows the socio-demographic data characteristics of the adult CF subjects.

\section{Depression in Adult CF Subjects}

Twenty CF subjects (17\%) were identified with HADS depression scores $\geq 8$. Out of these, 9 (45\%) CF subjects were suffering from both the anxiety and depressive symptoms. Five CF subjects (25\%) exhibited HADS depression scores $>11$ (potentially a case for clinical depression). A weak association was found between depression and male sex $(\mathrm{r}=0.17, P=.05)$, older age $(\mathrm{r}=0.18, P=.04)$, $\mathrm{FEV}_{1}(\mathrm{r}=-0.21, P=.03)$, living alone $(\mathrm{r}=-0.22$, $P=.03)$, and body mass index $(\mathrm{r}=-0.28, P<.01)$. A significant correlation was found between HAD depression and the total CF-QOL score $(\mathrm{r}=-0.72, P<.001)$ and hospital readmission score $(\mathrm{r}=0.40, P<.001)$.

Table 2 shows factors that had a significant independent association with depression in CF subjects. Quality of life measured by disease-specific CF-QOL, body mass index, and $\mathrm{FEV}_{1}$ were associated with depression. Age, sex, and number of comorbidities did not contribute to the regression model. Further analysis revealed that impaired quality of life measured by the CF-QOL score accounted for $23 \%$ of the variance (ie, those with poorer quality of life are 
Table 1. Demographic Characteristics $(n=121)$

\begin{tabular}{|c|c|}
\hline Age, mean $\pm \mathrm{SD}, \mathrm{y}$ & $30 \pm 8.8$ \\
\hline Body mass index, mean $\pm \mathrm{SD}, \mathrm{kg} / \mathrm{m}^{2}$ & $22 \pm 2.6$ \\
\hline Days of hospitalization, mean $\pm \mathrm{SD}$ & $10.8 \pm 18.3$ \\
\hline \multicolumn{2}{|c|}{$\begin{array}{l}\text { Cystic Fibrosis Quality of Life Scale Scores, } \\
\text { mean } \pm \text { SD }\end{array}$} \\
\hline Physical functioning & $80 \pm 17.9$ \\
\hline Social functioning & $81 \pm 21.9$ \\
\hline Treatment issues & $76.4 \pm 22.4$ \\
\hline Chest symptoms & $75 \pm 22.7$ \\
\hline Emotional functioning & $82 \pm 18.6$ \\
\hline Concerns about the future & $59 \pm 20.8$ \\
\hline Interpersonal relationships & $66 \pm 21$ \\
\hline Body image & $70 \pm 21$ \\
\hline Career concerns & $64 \pm 27.6$ \\
\hline Total score & $73 \pm 15.7$ \\
\hline Male/female, no. & $65 / 46$ \\
\hline \multicolumn{2}{|l|}{$\begin{array}{l}\text { Hospital Anxiety Depression Scale Score, } \\
\text { no. }(\%)\end{array}$} \\
\hline Depression 8-10 & $15(12.3)$ \\
\hline Depression $>11$ & $5(4.1)$ \\
\hline Anxiety $8-10$ & $27(22.3)$ \\
\hline Anxiety $>11$ & $13(11)$ \\
\hline \multicolumn{2}{|l|}{ Number of Comorbidities, no. (\%) } \\
\hline 0 & $30(25)$ \\
\hline $1-2$ & $40(33)$ \\
\hline $3-4$ & $36(30)$ \\
\hline$>5$ & $15(12)$ \\
\hline \multicolumn{2}{|c|}{$\begin{array}{l}\text { Frequency of Hospital Admission in Previous } \\
\text { Year, no. }(\%)\end{array}$} \\
\hline 0 & $73(60)$ \\
\hline 1 & $24(20)$ \\
\hline 2 & $16(13)$ \\
\hline$\geq 3$ & $8(7)$ \\
\hline
\end{tabular}

most likely to present with high level of depressive symptoms). Lower lung function accounted for $13 \%$ of the variance (ie, those patients with poorer lung function impairment are most likely to exhibit an elevated level of depressive symptoms), and lower BMI contributed to $8 \%$ of the variance (ie, those with lower BMI score are most likely to suffer with high level of depressive symptoms) in the adult CF patients with comorbid depression, respectively.

\section{Anxiety in Adult CF Subjects}

Forty CF subjects (33\%) were identified with HADS anxiety scores $\geq 8$. Out of these, $13(33 \%)$ of the subjects HADS anxiety scores $\geq 11$ (potentially a case for clinical anxiety). A statistically significant association was found between anxiety symptoms and interpersonal relationship ( $\mathrm{r}=-0.56, P<.001)$, chest symptoms $(\mathrm{r}=-0.47, P<.001)$, quality of life $(\mathrm{r}=-0.61, P<.001)$, age $(\mathrm{r}=0.21$, $P=.02)$, and hospital readmission $(\mathrm{r}=0.25, P=.01)$.

Table 3 shows findings of multiple regression analysis
Table 2. Factors That Are Related to Depression in Cystic Fibrosis Subjects (Multiple Regression Analysis)

\begin{tabular}{lcccc}
\hline \hline $\begin{array}{c}\text { Independent } \\
\text { Variable }\end{array}$ & $\begin{array}{c}\text { Standardized } \\
\text { Beta Value }\end{array}$ & T Value & $P$ & $\mathrm{r}^{2^{*}}$ \\
\hline Quality of life & -0.51 & -3.4 & $<.002$ & 0.23 \\
FEV $_{1}$ & -0.49 & -2.7 & .01 & 0.13 \\
Body mass index & -0.45 & 2.5 & .01 & 0.08 \\
& & & & \\
* Adjusted $\mathrm{r}^{2}=0.44$. & & & & \\
\hline
\end{tabular}

Table 3. Factors That Are Associated With Anxiety in Cystic Fibrosis Subjects (Multiple Regression Analysis)

\begin{tabular}{lcccc}
\hline \hline \multicolumn{1}{c}{$\begin{array}{c}\text { Independent } \\
\text { Variable }\end{array}$} & $\begin{array}{c}\text { Standardized } \\
\text { Beta Value }\end{array}$ & T Value & $P$ & $\mathrm{r}^{2^{*}}$ \\
\hline Interpersonal relationship & -0.42 & -4.21 & $<.001$ & 0.15 \\
Chest symptoms & -0.49 & -4.73 & $<.001$ & 0.09 \\
Age & 0.20 & 2.22 & .03 & 0.02 \\
& & & & \\
${\text { * Adjusted } \mathrm{r}^{2}=0.37}$ & & & & \\
\hline
\end{tabular}

with anxiety scores as the dependent variable. Factors that related with anxiety were interpersonal relationship score $(P<.001)$, frequent chest symptoms $(P<.001)$, and age $(P=.05)$. The full model accounted for $37 \%$ of the variance in the anxiety scores. Further data analysis showed $15 \%$ of the variance was accounted for by interpersonal relationship, which is the lower the score the poorer interpersonal relationship (the more anxious patients being more likely to have difficulty in their interpersonal relationship). Nine percent of the variance was accounted for by frequent chest symptoms (anxious patients are most likely to exhibit frequent chest symptoms (ie, lower score implies more frequent chest symptoms), and $2 \%$ of the variance by age (older patients being most likely to suffer with high level of anxiety).

\section{Health-Related Quality of Life in Relation to Anxiety and Depression}

Health-related quality of life measured by disease specific CF-QOL (physical functioning, social functioning, treatment issues, chest symptoms, emotional functioning, concerns for the future, interpersonal relationships, body image, future/career concerns, and total CF-QOL) were all significantly correlated with anxiety $(P<.001)$ and with depression $(P<.001)$, respectively.

Table 4 shows findings of the multiple regression analysis using the "total quality of life" score as a dependent variable. Anxiety, depression, and rehospitalization were significant independent predictors of quality of life in $\mathrm{CF}$ subjects. One percent of the variance was accounted for by 
Table 4. Variables That Are Related to Quality of Life (Multiple Regression Analysis)

\begin{tabular}{lccccc}
\hline \hline \multicolumn{1}{c}{$\begin{array}{c}\text { Independent } \\
\text { Variable }\end{array}$} & $\begin{array}{c}\text { Standardized } \\
\text { Beta Value }\end{array}$ & $\begin{array}{c}\mathrm{T} \\
\text { Value }\end{array}$ & $P$ & $\mathrm{r}^{2 *}$ \\
\hline $\begin{array}{l}\text { Anxiety on Hospital Anxiety } \\
\text { Depression scale }\end{array}$ & -0.25 & -2.90 & .005 & 0.16 \\
$\begin{array}{l}\text { Depression on Hospital Anxiety } \\
\quad \begin{array}{l}\text { Depression scale } \\
\text { Rehospitalization in previous year }\end{array}\end{array}$ & -0.52 & -5.70 & $<.001$ & 0.40 \\
& & & -1.85 & .06 & 0.01 \\
* Adjusted $\mathrm{r}^{2}=0.57$. & & & & & \\
\hline
\end{tabular}

episodes of readmission in the previous year (ie, those patients frequently admitted are most likely to experience poorer quality of life), $16 \%$ of the variance was accounted for by anxiety (ie, those with a high level of anxiety symptoms are more likely to exhibit impaired quality of life), and $40 \%$ of the variance was accounted for by depression (ie, those with high level of depressive symptoms are more likely to present with poorer health-related quality of life). The overall adjusted $\mathrm{R}^{2}=$ was 0.57 . Age, sex, body mass index, comorbidities, and lung function did not contribute to the model.

\section{Discussion}

The main findings of this study demonstrate that anxiety and depression are common in adult patients with CF. Depression and anxiety were associated with impaired quality of life and interpersonal relationships, rehospitalization, poorer lung function, older age, lower body mass index, and severity of respiratory symptoms.

In this study, about one in 3 CF subjects was suffering from a high level of clinically relevant anxiety symptoms, and about one in 6 with depressive symptoms. These elevated symptoms were higher in adult CF subjects attending the out-patient clinics than reported in a recent epidemiological survey, which found anxiety in one in $7 \mathrm{CF}$ patients, and depression in one in $10 .{ }^{7}$ There was a trend that adult patients ${ }^{7}$ with $\mathrm{CF}$ reported more elevated symptoms of anxiety than healthy control subjects. The reasons for potential discrepancies might be the latter data were collected over a 10-year period, which might be prone to potential health status changes and prevalence of anxiety and depressive symptoms may have fluctuated during this period. However, our point-of-prevalence rates of anxiety symptoms are slightly higher than previous single-center studies that reported the prevalence rates of anxiety symptoms ranging from 5\% to $27 \%, 5,6$ and the prevalence rates of depressive symptoms are comparable to previous studies between $10 \%$ to $17 \% .6-8$

\section{Association of Anxiety and Depression}

Quality of life measured by CF-QOL was associated with poor health status and accounted for $23 \%$ of the variability in the HADS depression score. This suggests that a CF-QOL score may provide invaluable information for clinicians about an individual's psychological functioning. However, the finding did not show the direction of causality; it is most likely the combined effect of respiratory impairment, physical disability, and severity of symptoms that may have contributed to impaired quality of life, in turn leading to poor psychological functioning. Havermans and colleagues ${ }^{6}$ found that, after controlling for lung function, depression (measured by the HADS scale) was associated with impaired quality of life. Low lung function was associated with depression in this study (after controlling for confounding variables age and sex). This finding was in agreement with previous studies ${ }^{5,6}$ that low lung function and impaired quality of life were associated with depression. However, caution is required in our interpretation of the finding that emotional functioning is one of the domains of quality of life that may have contributed for poorer psychological well-being. An alternative explanation could be that higher scores on quality of life result in maintenance of better psychological well-being.

Lower body mass index in adult CF patients was a minor predictor of depression. This suggests that patients who are depressed may lack the motivation and interest to prepare a balanced diet, which may require extra effort and organization. The alternative explanation might be that adult $\mathrm{CF}$ patients with severe lung function impairment and depression are less likely to eat with a 'full stomach' in fear of triggering dyspnea either at rest or on exertion. Therefore, adult CF patients who are underweight may benefit from having advice from a dietitian.

The fact that interpersonal relationships (relating to family member or friends) were negatively correlated with the high level of anxiety scores reflects that those having low scores are most likely to have difficulties in their relationships and were unable to cope with the impact of the disease. CF patients with a high level of anxiety may lack the motivation to engage in social and physical activities. ${ }^{5}$ Again, we cannot determine the direction of causality from these data. In a similar vein, chest symptoms scores (experiencing of more symptoms) were associated with a high level of anxiety symptoms. This implies that patients with frequent chest symptoms are most likely to be anxious and experience exacerbations, ${ }^{19}$ hospital readmission, and impaired quality of life. ${ }^{19,20}$

The association between impaired quality of life, anxiety and depression, and hospital readmission may reflect the complexity of coping with multiple illnesses and the subsequent consequences of hospital readmission. This suggests the importance of an integrated disease management 
approach that includes counseling and psycho-educative plans such as written information on how individual $\mathrm{CF}$ patients self-manage their comorbid anxiety and depressive symptoms. Untreated anxiety and depressive symptoms may have deleterious effect on psychological wellbeing, such as feelings of hopelessness and loneliness. Previous studies ${ }^{6-8}$ have shown that younger age was associated with impaired quality of life and high level of anxiety and depressive symptoms. Indeed, these factors may compromise more the coping strategies of younger CF patients, compared to older CF patients, especially during exacerbations. Episodes of readmission had a moderate association with impaired quality of life in this subgroup of the adult CF population. This perhaps may be explained by the fact that frequent hospitalization potentially dislocates the social relationships and routine work related daily activities, which might be more frustrating and upsetting for the patients. However, we have not explored the cause(s) or factors that contribute to hospital readmission. Therefore, caution is regarded in the interpretation of our findings.

There are several limitations to this study. First, this is a cross-sectional study in one large referral center, without a control group. Our findings are limited in terms of exploring the incidence and recovery of anxiety and depressive symptoms. During the study period we managed to recruit $40 \%$ of the sample of those eligible CF patients (approximately 300) who were in the registry list in the center. We were unable to confirm or refute whether nonparticipants differed from participants in terms of demographic characteristics or variables of interest. Second, although several factors have been reported to be predictors of anxiety and depression, the causal relationship cannot be assumed from these data. Third, the sample size is relatively adequate for the variables we have examined. However, the cross-sectional nature of the design does not tell us any inferences in the direction of causality (eg, between depression and lung function impairment). Thus, replication of our findings with a larger sample size in a longitudinal multi-center study is worthy of endeavor. Fourth, we used a self-reported HADS scale to assess anxiety and depressive symptoms. Therefore, we have not used clinical interviews, for example, SCID (Structured Clinical Interviews Diagnostic), to confirm or refute the diagnoses of major clinical depression in those CF subjects with elevated anxiety and depressive symptoms (eg, HADS depression or anxiety $\geq 8$ ). Fifth, we carried out multiple testing to examine the relationship between psychological, quality of life, and physiological (lung function) variables. We have attempted to reduce the variability by adjustment for multiple comparisons. In addition, there are very few or no missing data in the variables collected for the data analysis. The limitation of the multiple regression analysis is that it may have introduced type 1 error. ${ }^{18,21}$ However, in this exploratory study, the derivation of a definitive set of predictors is not the main objective.

\section{Clinical and Research Implications}

Undiagnosed anxiety and depressive symptoms are common in adult patients with CF. They are associated with impaired quality of life and interpersonal difficulties, frequent hospital readmission, older age, lower body mass index, and severity of respiratory symptoms. We advocate that CF patients be routinely monitored for anxiety and depressive symptoms, and those patients who exhibit high level of symptoms should be treated with individually tailored pharmacological (antidepressants) and non-pharmacological treatment approaches (eg, home based rehabilitation). Therefore, identifying and treating depression and anxiety in patients with CF may help to improve quality of life and reduce healthcare utilization. Currently, there are no controlled clinical trials that have investigated the efficacy of antidepressant drug therapy or psychological therapy (eg, cognitive behavioral therapy) in the management of these comorbid disorders in CF patients. Future studies are warranted.

\section{Conclusions}

In summary, our findings indicate that anxiety and depressive symptoms are common in CF patients. Untreated anxiety and depression have substantial impact on $\mathrm{CF}$ patients' quality of life, physical function, and healthcare utilization. Routine screening for symptoms of anxiety and depression is a worthy endeavor, and those identified with elevated clinical symptoms should be referred to receive appropriate treatment.

\section{ACKNOWLEDGMENTS}

We are most grateful to Ms Helen Oxley, Manchester Adult Cystic Fibrosis Centre, Wythenshawe Hospital, Manchester, United Kingdom, for her support during the data collection. We are also most grateful to Ms Julie Morris, Department of Biostatistics, School of Medicine, University of Manchester, Manchester, United Kingdom, for her support and expert advice in the data analysis.

\section{REFERENCES}

1. Bobadilla JL, Macek M Jr, Fine JP, Farrell PM. Cystic fibrosis: a worldwide analysis of CFTR mutations-correlation with incidence data and application to screening. Hum Mutat 2002;19(6):575-606.

2. Farrell PM. Improving the health of patients with cystic fibrosis through newborn screening. Wisconsin Cystic Fibrosis Neonatal Screening Study. Adv Ped 200;47(1):79-115.

3. Sims EJ, Mugford M, Clark A, Aitken D, McComrick J, Mehta G, Mehta A; UK Cystic Fibrosis Database Steering Committee. Economic implications of newborn screening for cystic fibrosis: a cost of 
illness retrospective cohort study. Lancet 2007;369(9568):1187-1195. Erratum in: Lancet 2007;370(9581):28.

4. de Jong W, van der Schans CP, Mannes GP, van Aalderen WM, Grevink RG, Koeter GH. Relationship between dyspnoea, pulmonary function and exercise capacity in patients with cystic fibrosis. Respir Med 1997;91(1):41-46.

5. Epker J, Maddrey JM. Quality of life in pediatric patients with cystic fibrosis. Int J Rehabil Health 1998;4(5):215-222.

6. Havermans T, Colpart K, Dupont LJ, Quality of life in patients with cystic fibrosis: association with anxiety and depression. J Cyst Fibrosis 2008;7(6):581-584.

7. Goldbeck LT, Besier, T, Hins A, Singers S, Quittner AL; the TIDES Group. Prevalence of anxious and depressive symptoms in German patients with cystic fibrosis. Chest 2010;138(4):929-936.

8. Riekert KA, Bartlett SJ, Boyle MP, Krishnan JA, Rand CS. The association between depression, lung function, and health-related quality of life among adults with cystic fibrosis. Chest 2007;132(7):231-237.

9. Gee L, Abbott J, Conway S, Etherington C, Webb AK. Development of a disease specific health related quality of life measure for adults and adolescents with cystic fibrosis. Thorax 2000;55(11):946-954.

10. Quittner AL, Sweeny S, Watrous M, Munzenberger P, Bearss K, Gibson NA, et al. Translation and linguistic validation of a diseasespecific quality of life measure for cystic fibrosis. J Pediatr Psychol 2000;25(6):403-414.

11. Gee L, Abbott J, Conway S, Etherington C, Webb AK. Quality of life in cystic fibrosis: the impact of gender, general health perceptions and disease severity. J Cyst Fibrosis 2003;2(4):206-213.
12. Cruz I, Marciel KK, Quittner AL, Schechter MS. Anxiety and depression in cystic fibrosis. Semin Respir Crit Care Med 2009;30(5):569-578.

13. Goss $\mathrm{CH}$, Burns JL. Exacerbations in cystic fibrosis. 1: epidemiology and pathogenesis. Thorax 2007;62(4):360-367.

14. Rabe KF, Hurd S, Anzuteo A, Barnes PJ, Buist S, Calverley P, et al. Global strategy for the diagnosis, management, and prevention of chronic obstructive pulmonary disease: GOLD executive summary. Am J Respir Crit Care Med 2007;176(6):532-555.

15. Zigmond AS, Snaith RP. The hospital anxiety and depression scale. Acta Psychiatr Scand 1983;67(6):361-370.

16. Bjelland I, Dahl AA, Haug TT, Necklmann D. The validity of the hospital anxiety and depression scale: an updated the literature reviews. J Psychosom Res 2002;52(2):69-77.

17. Cameron IM, Crawford JR, Lawton K, Reid IC. Psychometric comparison of PHQ-9 and HADS for measuring depression severity in primary care. Br J Gen Pract 2008;58(546):32-36.

18. Tabachnick BG, Fidell LS, Using multivariate statistics, 3rd edition. New York: Happer Collins; 1996.

19. Britto MT, Kotagal UR, Hormung RW, Tsevat J, Wilmott RW. Impact of recent exacerbations on quality of life in patients with cystic fibrosis. Chest 2002;121(1):64-72.

20. Gee L, Abbott J, Hart A, Conway SP, Etherington C, Webb AK. Associations between clinical variables and quality of life in adults with cystic fibrosis. J Cyst Fibrosis 2005;4(1):59-66.

21. Greenland S. Variables selection versus shrinkage in the control of multiple confounders. Am J Epidemiology 2008;167(5):523-529. 